Research Article

\title{
Chronic Intermittent Hypoxia Induces the Long-Term Facilitation of Genioglossus Corticomotor Activity
}

\author{
Ying Zou $\left(\mathbb{D}\right.$, Wei Wang $\mathbb{D}^{\mathbb{D}}$, Xinshi Nie, and Jian Kang \\ Institute of Respiratory Disease, The First Hospital of China Medical University, Shenyang, China \\ Correspondence should be addressed to Wei Wang; wwbycmu@126.com
}

Received 23 October 2017; Revised 21 February 2018; Accepted 12 March 2018; Published 23 April 2018

Academic Editor: Rocco Trisolini

Copyright (c) 2018 Ying Zou et al. This is an open access article distributed under the Creative Commons Attribution License, which permits unrestricted use, distribution, and reproduction in any medium, provided the original work is properly cited.

\begin{abstract}
Obstructive sleep apnea (OSA) is characterized by the repetitive collapse of the upper airway and chronic intermittent hypoxia $(\mathrm{CIH})$ during sleep. It has been reported that $\mathrm{CIH}$ can increase the EMG activity of genioglossus in rats, which may be related to the neuromuscular compensation of OSA patients. This study aimed to explore whether $\mathrm{CIH}$ could induce the long-term facilitation (LTF) of genioglossus corticomotor activity. 16 rats were divided into the air group $(n=8)$ and the CIH group ( $n=8)$. The CIH group was exposed to hypoxia for 4 weeks; the air group was subjected to air under identical experimental conditions in parallel. Transcranial magnetic stimulation (TMS) was applied every ten minutes and lasted for $1 \mathrm{~h} /$ day on the 1st, 3rd, 7th, 14th, 21st, and 28th days of air/CIH exposure. Genioglossus EMG was also recorded at the same time. Compared with the air group, the $\mathrm{CIH}$ group showed decreased TMS latency from 10 to 60 minutes on the 7th, 14th, 21st, and 28th days. The increased TMS amplitude lasting for 60 minutes was only observed on the 21st day. Genioglossus EMG activity increased only on the 28th day of $\mathrm{CIH}$. We concluded that CIH could induce LTF of genioglossus corticomotor activity in rats.
\end{abstract}

\section{Introduction}

Obstructive sleep apnea syndrome (OSAS) is mainly manifested as the recurrent collapse of the upper airway during sleep. Considering that the intermittent hypoxia $(\mathrm{IH})$ is a hallmark of sleep apnea, the IH-induced long-term facilitation (LTF) was an intriguing finding [1]. As the most common model of respiratory plasticity, LTF is characterized by an escalation of the respiratory motor activation during the normoxia period following the intermittent hypoxia and by a long-lasting enhancement in respiratory activity for 90 minutes after repetitive IH exposure [2]. LTF, as a kind of plasticity of respiratory motoneuron activity, has many forms including phrenic nerve activity LTF (pLTF), hypoglossal nerve activity LTF (hLTF), ventilation LTF (vLTF), and sensory LTF (sLTF) [3]. It was postulated that the exposure to IH throughout the night might elicit the LTF of respiratory and upper airway muscle activities, which could mitigate the cyclical events characterized by breathing instability. Indeed, Mckay et al. observed that episodic hypoxia evoked the LTF of genioglossus EMG in newborn rats, indicating that genioglossus LTF played an important role in preventing the upper airway from collapse [4]. Mateika et al. reported that the exposure to mild $\mathrm{IH}$ resulted in a reduction in the therapeutic continuous positive airway pressure that required eliminating breathing events [5]. Thus, it is reasonable to assume that upper airway muscle LTF might be a protective mechanism against apnea. This has also been confirmed by Powell and Mitchell, who observed that ventilatory LTF could maintain respiratory stability especially during sleep [6]. Nevertheless, the occurrence of LTF at the level of the genioglossus corticomotor area remains unknown, especially during and after the daily exposure to $\mathrm{CIH}$. It could be assumed that this $\mathrm{IH}$-induced plasticity might present as a dynamic alteration of the corticomotor excitability and the EMG activity of genioglossus over time.

Transcranial magnetic stimulation (TMS) technique can be used to explore the cortical spinal cord conduction pathway of the skeletal muscle. With the technique of transcranial magnetic stimulation (TMS), Wang et al. studied the TMS response of the genioglossus corticomotor area in normal and OSAS patients $[7,8]$. Sériès et al. further studied 
genioglossus corticomotor activity in awake OSAS patients and observed negative correlation between the apneahypopnea index (AHI) and TMS latency [8]. Our previous study also found an increased activity of the genioglossus corticomotor area in the rats during 4 weeks of $\mathrm{CIH}$ [9]. These above findings suggested that OSAS patients had central compensation during wakefulness, which might be related to the exposure to $\mathrm{CIH}$. In this regard, this study aimed to explore the alteration of genioglossus corticomotor excitability using TMS and its EMG activity over time after $\mathrm{IH}$ stimulation at different stages of $\mathrm{CIH}$ in rats.

\section{Animals and Materials}

2.1. Animals. Sixteen adult male Wistar rats $(280-300 \mathrm{~g}$, 8 weeks old) were provided by Liaoning Changsheng Biotechnology Company (Benxi City, China). The rats were randomly divided into two groups: the $\mathrm{CIH}$ group $(n=8)$ and the air group $(n=8)$. All animals were housed in polypropylene cages with a capacity of $15 \mathrm{~cm} \times 20 \mathrm{~cm} \times 20 \mathrm{~cm}$, were given free access to water and food, and were housed under controlled conditions (temperature $24 \pm 2^{\circ} \mathrm{C}$ and relative air humidity $40 \%$ ) with a $12: 12 \mathrm{~h}$ light-dark cycle (lights on at 8:00 am and lights off at 8:00 pm). All procedures were performed in accordance with the National Institutes of Health Guide for Care and Use of Laboratory Animals and were approved by the Animal Ethics and Use Committee of China Medical University.

2.2. Chronic Intermittent Hypoxia. The rats in the $\mathrm{CIH}$ group were subjected to oxycycler (OxyCycler model A84XOV; BioSpherix, NY) hypoxia $\left(10 \% \mathrm{O}_{2}\right.$ in $\mathrm{N}_{2}$ for $\left.45 \mathrm{~s}\right)$ and normoxia $\left(21 \% \mathrm{O}_{2}\right.$ in $\mathrm{N}_{2}$ for $72 \mathrm{~s}$ ) every $180 \mathrm{~s}$ for $8 \mathrm{~h} / \mathrm{d}$ (from 8 am to $4 \mathrm{pm}$ ), for 4 consecutive weeks. The rats in the air group were subjected to air under identical experimental conditions in parallel. The $\mathrm{O}_{2}$ concentration was continuously measured by an $\mathrm{O}_{2}$ analyzer and was changed by a computerized system controlling the gas outlets (shown as the Supplementary Figure 2). On the 1st, 3rd, 7th, 14th, 21st, and 28th days of the experiments in all groups, the last $30 \mathrm{~min}$ could be considered as acute intermittent hypoxia stimulation, as previously mentioned [10].

\subsection{Transcranial Magnetic Stimulation (TMS). Transcranial} magnetic stimulation was carried out on the $1 \mathrm{st}, 3 \mathrm{rd}, 7 \mathrm{th}$, 14th, 21st, and 28th days of the experiments in all groups. The rats were supinely positioned on a wooden board, and their heads, bodies, and limbs were restrained. Then, singlepulse TMS was performed by a Magstim 200 stimulator (Magstim, Whiteland, Dyfed, UK) with a $25 \mathrm{~mm}$ figure-eight coil. The coil was held against the rat's head. The TMS response of the genioglossus corticomotor area was described in terms of the corresponding motor-evoked potential (MEP) amplitude and latency. The optimal coil position was defined as that with the best response to TMS (the highest MEP amplitude and the shortest MEP latency). Briefly, the optimal stimulation site was $3.0-5.0 \mathrm{~mm}$ rostral to bregma and $2.0-$ $4.0 \mathrm{~mm}$ lateral from the midline (shown as the supplementary
Figure 1). The site was clearly marked with indelible ink, and the position of the coil was kept constant at the stimulation site by using a high-precision multipositional support consisting of two articulated arms. For each position, three stimuli were applied with at least $30 \mathrm{~s}$ interval and averaged for the mean MEP response. All TMSs were delivered at the end of normal expiration in the rats after intermittent hypoxia. The respiratory phase was determined by the detection of abdominal movement [9]. A concentric needle electrode (NM-131 T, Nihon Kohden, Japan) was inserted into the genioglossus. MEP was recorded using a computer software package (AxoScope software 9.0, Axon Instruments, Inc., USA). MEP latency was defined as the time up to the first deflection from baseline following TMS, and its amplitude was measured from the peak-to-peak TMS response. The latency and amplitude of the TMS response were recorded at $10 \mathrm{~min}, 20 \mathrm{~min}, 30 \mathrm{~min}, 40 \mathrm{~min}$, $50 \mathrm{~min}$, and $60 \mathrm{~min}$ after intermittent hypoxia, respectively, on each day (Supplementary Tables 1-3). The rats were appropriately anesthetized with sodium pentobarbital $(40 \mathrm{mg} / \mathrm{kg})$. The methodology is described in detail in our previous studies $[9,11]$.

2.4. Genioglossus EMG. The EMG activity of genioglossus was recorded by inserting a concentric needle electrode (NM-131T, Nihon Kohden, Japan) into the genioglossus. It was filtered $(300-10,000 \mathrm{~Hz})$, rectified, and integrated (Paynter Filter, BAK Electronics, Mount Airy, MD; time constant $200 \mathrm{~ms}$ ). The integrated signals were digitized and acquired with computer software (LabVIEW 8.0, National Instruments) and analyzed with a customized program $[3,10]$. This software determined the amplitude and timing of integrated EMG activity during early inspiration, from which the EMG of genioglossus was calculated. Genioglossus EMG was recorded at $10 \mathrm{~min}, 20 \mathrm{~min}, 30 \mathrm{~min}, 40 \mathrm{~min}$, $50 \mathrm{~min}$, and $60 \mathrm{~min}$ after the exposure to intermittent hypoxia, respectively, on each day.

Cardiorespiratory parameters (heart rate and central respiratory rate) were calculated over $10 \mathrm{~min}$ interval prior to the performance of TMS. If needed, supplemental doses of sodium pentobarbital were administered as increments until the absence of a toe-pitch withdrawal reflex.

2.5. Statistics Analysis. The results were reported as means \pm SD. For the analysis of genioglossus EMG activities and TMS responses among different groups at the same time point, a repeated measure two-way ANOVA followed by Dunnett's studentized test was applied with two factors: times and treatments. All data were analyzed using SPSS 17.0 software. $p<0.05$ was considered statistically significant.

\section{Results}

Cardiorespiratory parameters (heart rate and central respiratory rate) had no significance difference from $10 \mathrm{~min}$ to 60 min during the experiment.

3.1. EMG. Compared with the baseline value, the $\mathrm{CIH}$ group begun to show an increased EMG activity of the genioglossus 
at 10 and 20 min after hypoxia stimulation on the 14th day (71.63 \pm 7.41 versus $82.62 \pm 11.48$ and $84 \pm 8.29$ for values at baseline, $10 \mathrm{~min}$, and $20 \mathrm{~min}$ after $\mathrm{IH}$, resp.; $p<0.05$ ). Compared with the air group, the $\mathrm{CIH}$ group revealed the increased EMG activity of the genioglossus at $10 \mathrm{~min}$ and $20 \mathrm{~min}$ after hypoxia stimulation on the 14th day $(75.78 \pm$ 2.34 versus $82.62 \pm 11.48$ and $74.77 \pm 1.08$ versus $84 \pm 8.29$ for values at $10 \mathrm{~min}$ and $20 \mathrm{~min}$ after IH for the air and $\mathrm{CIH}$ groups, resp.; $p<0.05)$. This increment of EMG activity persisted within $30 \mathrm{~min}$ after the $\mathrm{IH}$, as observed by a decline of the value at $30 \mathrm{~min}$. At the 28th day, the increased genioglossus EMG activity lasted for $60 \mathrm{~min}$ (Figure 1).

3.2. TMS Responses. The value of genioglossus latency detected by TMS was highly reproducible, with an average coefficient of variation of 5.5\%. In terms of TMS latency recorded in the $\mathrm{CIH}$ group, it significantly decreased from 10 to $30 \mathrm{~min}$ after hypoxia stimulation on the 1st day of $\mathrm{CIH}$, when compared with the baseline value $(4.46 \pm 0.01,4.64 \pm 0.15$, $4.71 \pm 0.05$, and $5.03 \pm 0.05$ for $10,20,30 \mathrm{~min}$, and baseline value, resp.; $p<0.05)$ and the air group $(4.46 \pm 0.01$ versus $5.13 \pm 0.09,4.64 \pm 0.15$ versus $5.14 \pm 0.11$, and $4.71 \pm 0.05$ versus $5.14 \pm 0.08$ for 10,20 , and $30 \mathrm{~min}$, resp.; $p<0.05$ ). At the 7 th day of $\mathrm{CIH}$, TMS latency decreased from $10 \mathrm{~min}$ after hypoxia and this decrement lasted for the following $50 \mathrm{~min}$. This persistence of the decrement of latency was observed for the following 3 weeks (Figure 2). Besides, TMS latency reached the valley point $(4.37 \pm 0.09)$ at $10 \mathrm{~min}$ after hypoxia on the $21 \mathrm{st}$ day of $\mathrm{CIH}$. There was no statistical difference of the value detected at $60 \mathrm{~min}$ among each day for TMS latency. However, TMS amplitude only showed LTF at the 21st day of $\mathrm{CIH}$ (Figure 3).

In terms of TMS amplitude, the difference between the air and $\mathrm{CIH}$ groups was only observed from $10 \mathrm{~min}$ to $60 \mathrm{~min}$ after IH on the 21 st day (60 min; $p=0.023$ ).

\section{Discussion}

This study was designed to explore the LTF of genioglossus EMG and its corticomotor activity at different stages of $\mathrm{CIH}$ in rats. We observed that a decreased TMS latency lasted for 60 minutes after the IH from the 7th day to the 28th day in the $\mathrm{CIH}$ group, when compared with the air group. Meanwhile, the persistent increase of the TMS amplitude and the genioglossus EMG activity from 10 to $60 \mathrm{~min}$ after the $\mathrm{IH}$ was only observed on the 21 st and 28 th days of $\mathrm{CIH}$, respectively. These results indicated that $\mathrm{CIH}$ could induce the LTF of genioglossus corticomotor activity, which occurs prior to the facilitation of the excitability of genioglossus muscle activity in rats.

As a special respiratory muscle, the alteration of the activity of genioglossus during $\mathrm{CIH}$ was of great concern because it plays an important role in exploring the pathogenesis and treatment of OSAS. In 2004, McKay et al. put the newborn rats to episodic hypoxia (5\% oxygen for $5 \mathrm{~min}$ and three cycles) and found that genioglossus EMG progressively increased and lasted for $60 \mathrm{~min}$ after $\mathrm{CIH} \mathrm{[4].} \mathrm{Tu} \mathrm{et} \mathrm{al.}$ observed the LTF of the discharge of the hypoglossal nerve after 4 weeks of $\mathrm{CIH}$ in anesthetized and vagotomized rats $[3,10]$. Based on these findings, the present study further explored the change of genioglossus at the central control level and confirmed that CIH could evoke the LTF of genioglossus corticomotor activity. This augmented corticomotor excitability presented as early as the first week of $\mathrm{CIH}$ and persisted throughout the following 3 weeks of $\mathrm{CIH}$. On the other hand, the increment of genioglossus EMG activity has not been observed until the 4 th week of $\mathrm{CIH}$. These indicated that $\mathrm{CIH}$ could induce the enhanced corticomotor excitability of genioglossus at the early stage of $\mathrm{CIH}$, and this central compensation occurs prior to the augmented genioglossus activity. This study was the first to continuously observe the LTF of genioglossus EMG activity and its associated central control during $\mathrm{CIH}$ in rats.

The fact that the recurrent obstructive sleep apneas occur only during sleep suggests that OSAS patients may have neuromuscular compensation of the upper airway during wakefulness [12]. Recently, two unexpected benefits of $\mathrm{IH}$ had been recognized as to improve respiratory and nonrespiratory somatic motor functions and to increase growth/trophic factor expression in the central nervous system. It was well known that CIH could induce the LTF of not only ventilation but also respiratory muscle activity $[13,14]$. Hu et al. reported that 4 weeks of CIH could lead to a steady increase of genioglossus EMG activity [15]. Klawe et al. also found that snorers and OSA patients presented higher genioglossus activity with progressive hypoxia [13]. What is the underlying mechanism associated with this $\mathrm{CIH}$-induced alteration of genioglossus activity? Besides, it remains unknown whether the excitatory inputs to genioglossus are derived from the central control. Moss et al. had reported that when exposed to recurrent episodic hypoxia, neonates expressed a tolerance to a subsequent hypoxic stimulus with relative hypoventilation; this change might relate to an adaptation of the respiratory center, and this adjustment made in response to repeated hypoxic episodes is assumed to be advantageous to the subject [14]. Therefore, we assumed that the $\mathrm{CIH}$-induced central compensation might occur prior to the facilitation of the excitability of the genioglossus EMG activity. In the present study, the observation that the occurrence of LTF at different levels was presented in an order of priority has confirmed our hypothesis. Moreover, this study showed that the increment of genioglossus corticomotor activity occurred rapidly after the exposure to $\mathrm{CIH}$, and this augmented excitability persisted throughout the early stage of $\mathrm{CIH}$.

Although the mechanisms underlying OSA are complex, there is general agreement that a sleep-related decline in upper airway muscle activity contributes to airway narrowing and/or collapse [16]. Compared with normal subjects, OSAS patients showed increased GG EMG activity during wakefulness, while it decreased during sleep [12]. Many scholars tried to strengthen the mechanical performance of genioglossus, the contraction of which could counterbalance the anatomically unfavorable and collapsible upper airway in OSA patients. Mwenge et al. reported that the electrical stimulation, by implanted electrodes in the sublingual nerve, could decrease the apnea-hypopnea index 


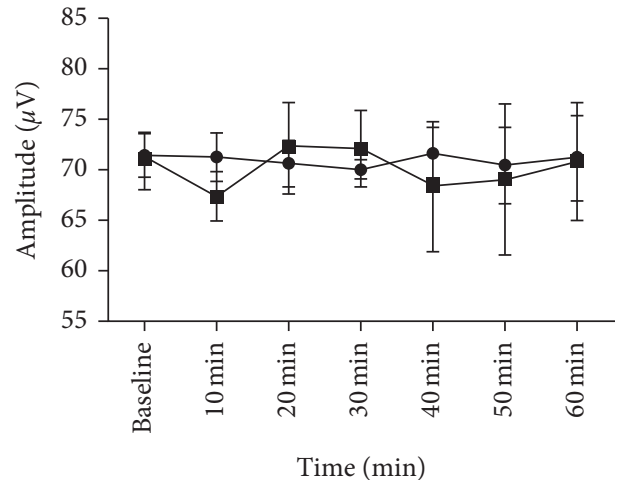

- $\mathrm{CIH}$ exposure on 1st day

- Air exposure on 1st day

(a)

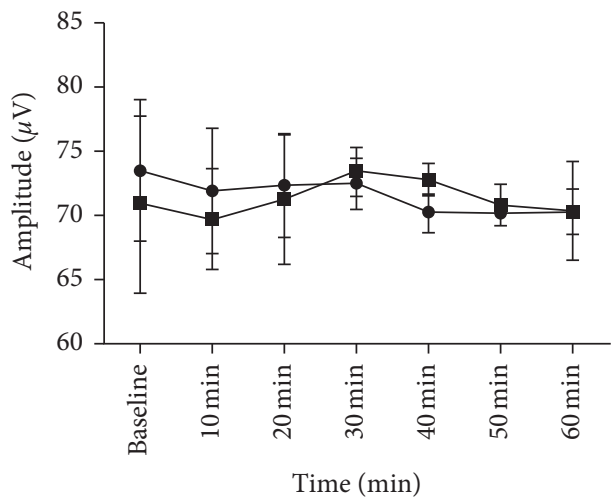

- $\mathrm{CIH}$ exposure on 7th day

- Air exposure on 7th day

(c)

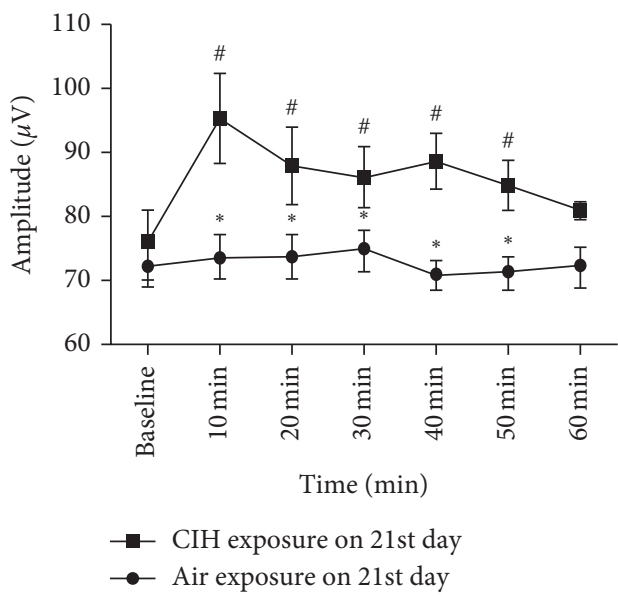

(e)

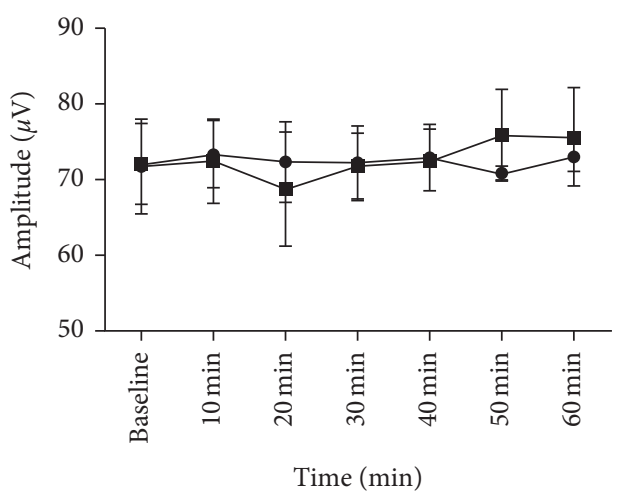

$\mathrm{CIH}$ exposure on 3rd day

Air exposure on 3rd day

(b)

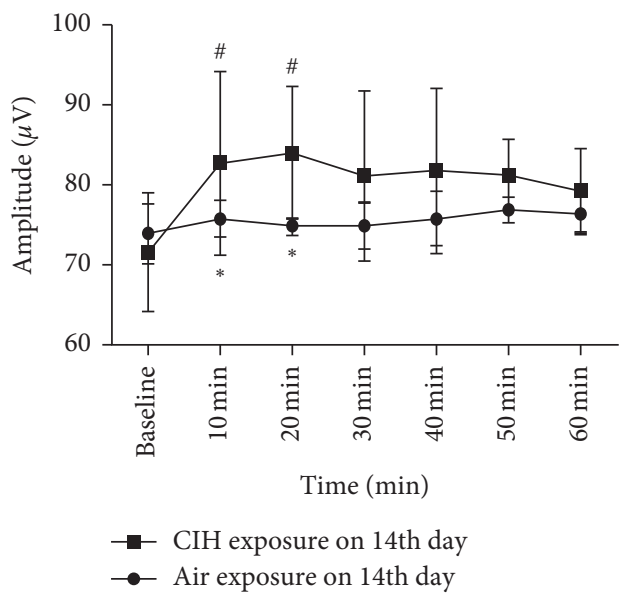

(d)

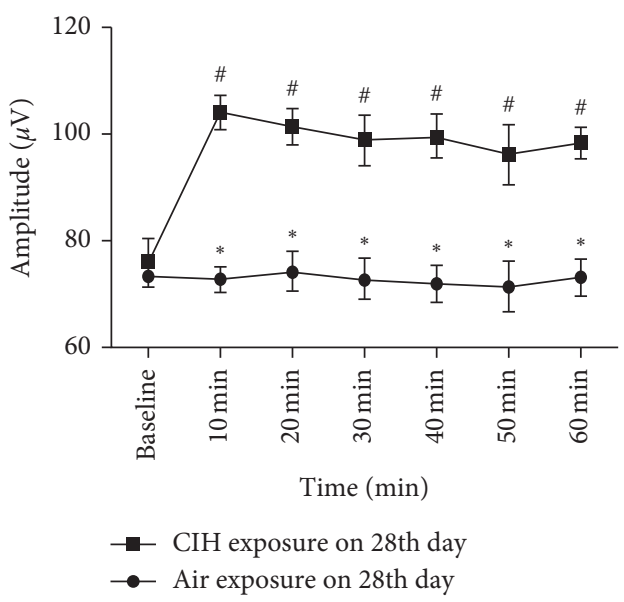

(f)

Figure 1: The comparison of genioglossus EMG activity at different time points after IH stimulation among different groups. (a-f) Values obtained on the 1st, 3rd, 7th, 14th, 21st, and 28th days of the daily air/CIH exposure. ${ }^{*}$ Difference between AIR and CIH groups at the same time point. "Difference between values detected at different time points in the CIH group.

(AHI) in OSAS patients [17]. Behan et al. also applied the approach of tongue exercise to increase the serotonin excitability inputs into the hypoglossal nerve, which innervate the contraction of genioglossus [18]. Furthermore, the LTF of neural drive to upper airway muscles during sleep could minimize or prevent apneic events [19]. Consistent with this suggestion, hypoxic episodes in sleeping OSA patients could induce an upregulation of the genioglossus corticomotor excitability, which could possibly facilitate the contraction of the genioglossus and the maintenance of UA patency. 


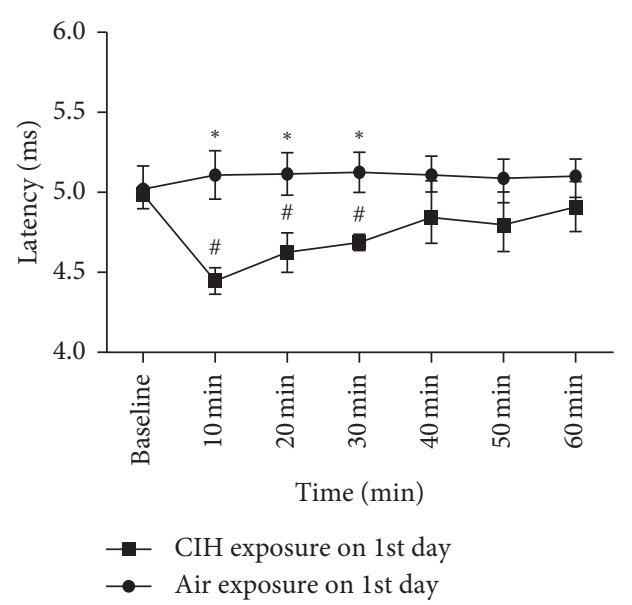

(a)

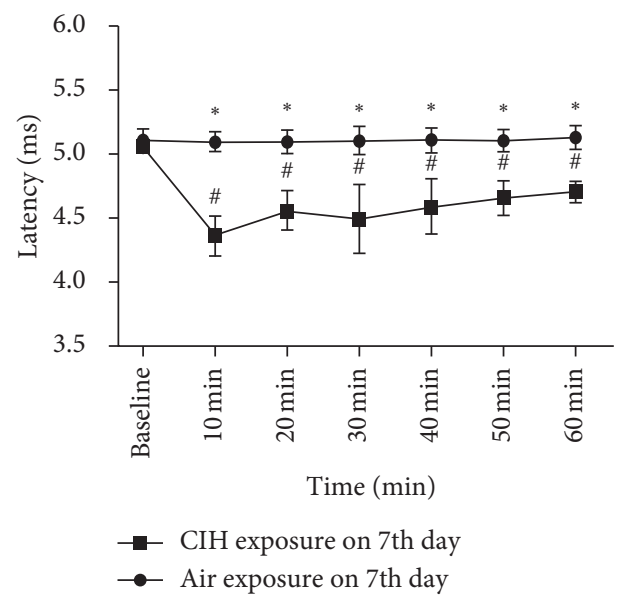

(c)

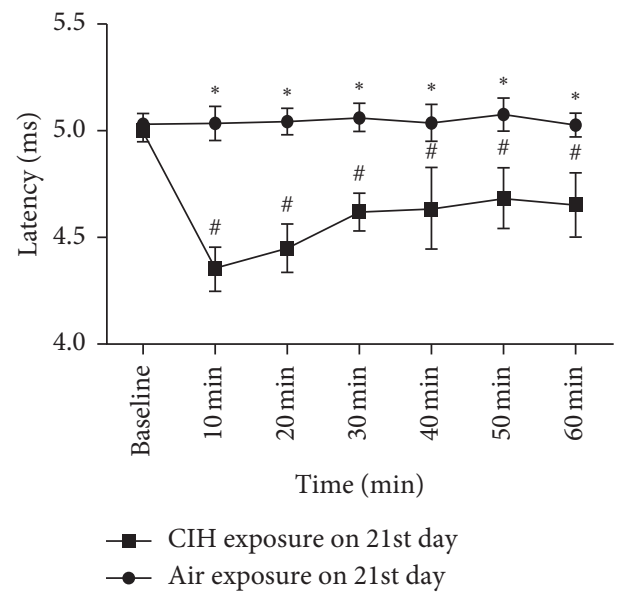

(e)

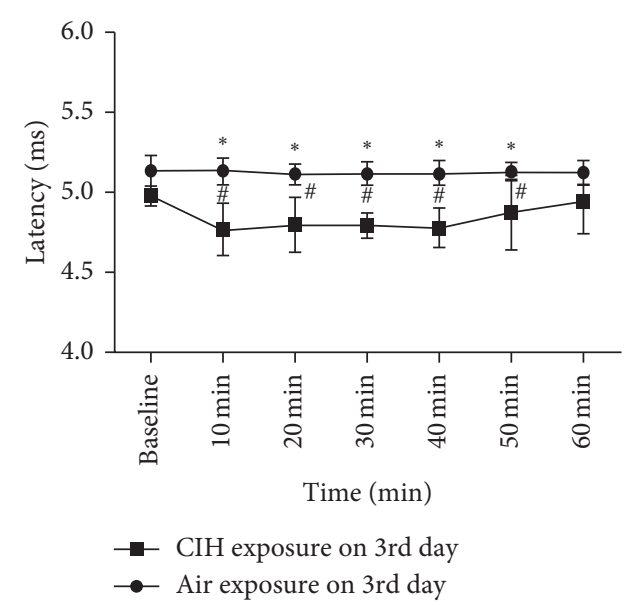

(b)

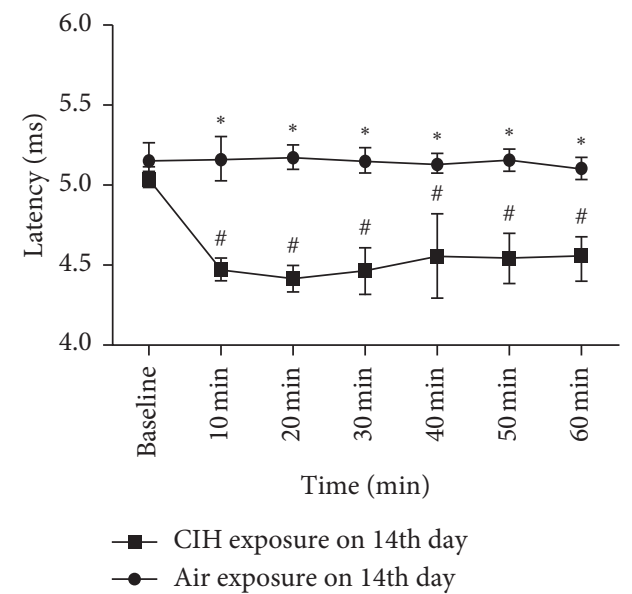

(d)

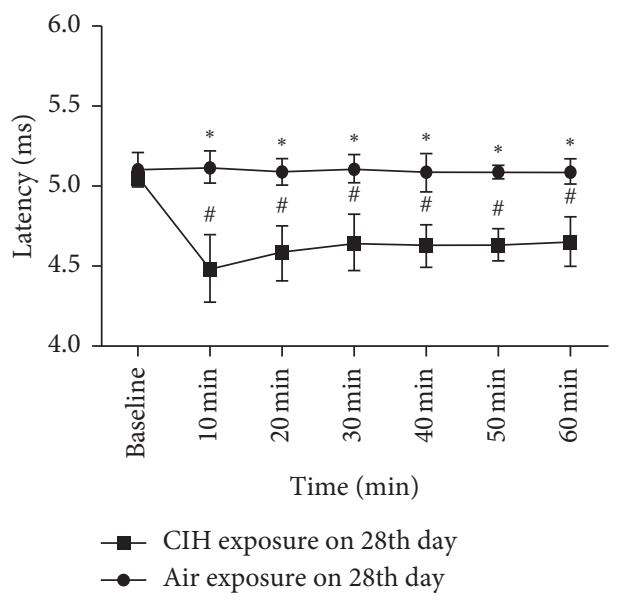

(f)

FIgURE 2: The comparison of TMS latency at different time points after IH stimulation among different groups. (a-f) Values obtained on the $1 \mathrm{st}, 3 \mathrm{rd}, 7 \mathrm{th}, 14 \mathrm{th}, 21 \mathrm{st}$, and $28 \mathrm{th}$ days of the daily air/CIH exposure. ${ }^{*}$ Difference between AIR and CIH groups at the same time point. ${ }^{\#}$ Difference at different time points on each day in the $\mathrm{CIH}$ group.

Nevertheless, whether this upregulation of the genioglossus corticomotor excitability transfers into the promotion of the pharyngeal airway stability during sleep deserves to be further investigated.
The limitation of this study was listed as follows: firstly, it is regretting that we did not observe the LTF of genioglossus and its central control during sleep. In our previous studies, the central compensation was observed only during wakefulness 


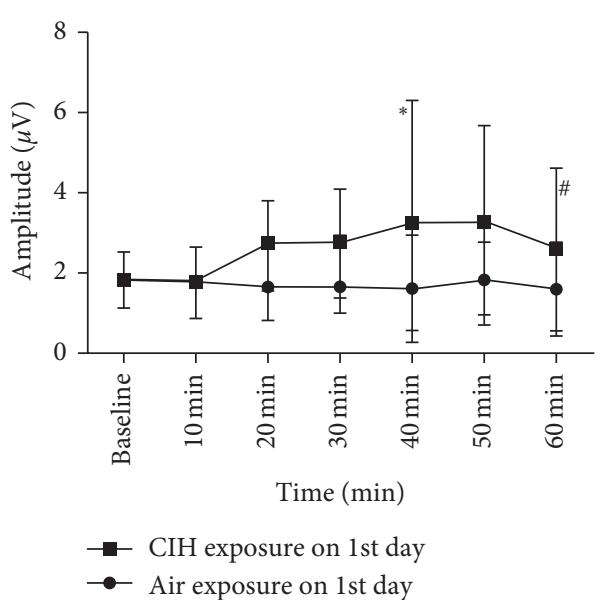

(a)

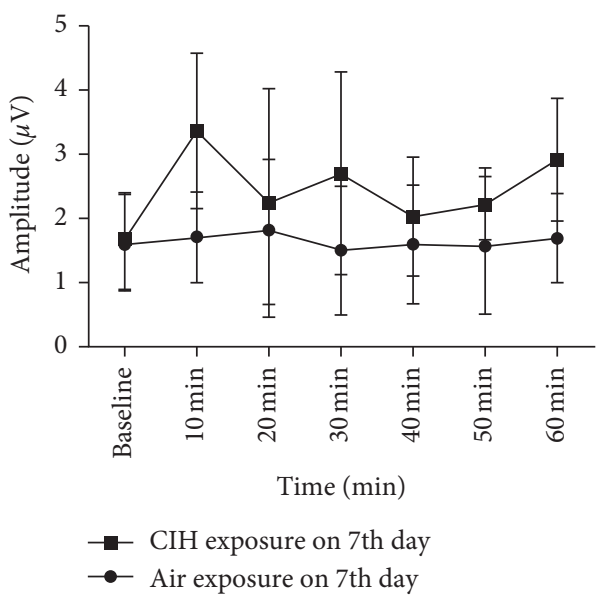

(c)

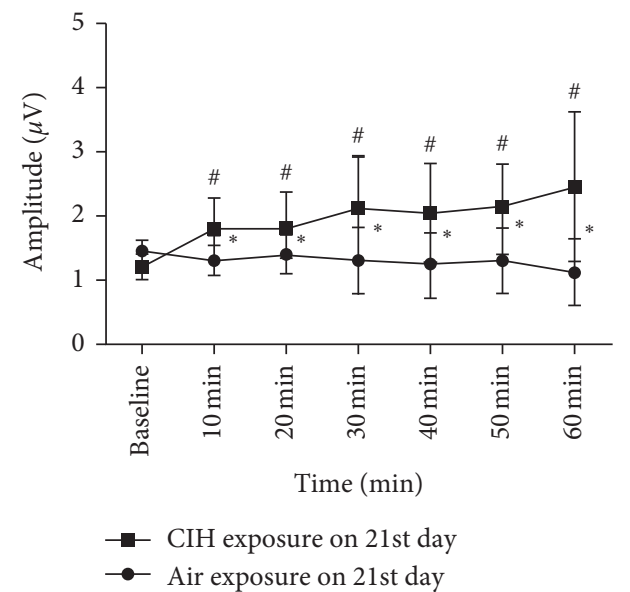

(e)

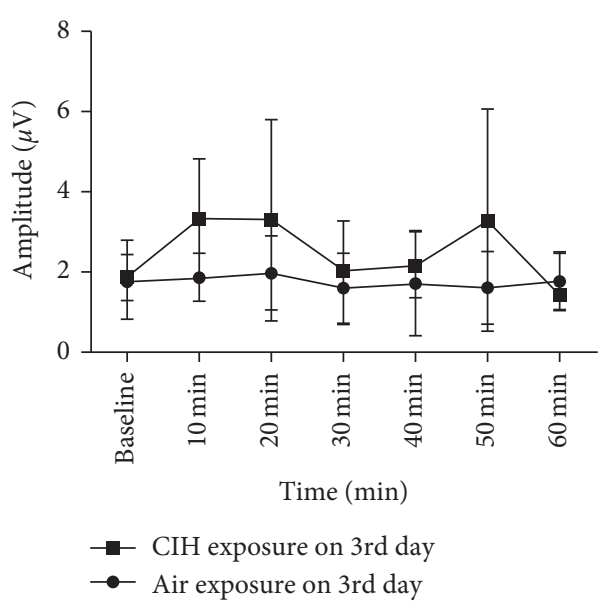

(b)

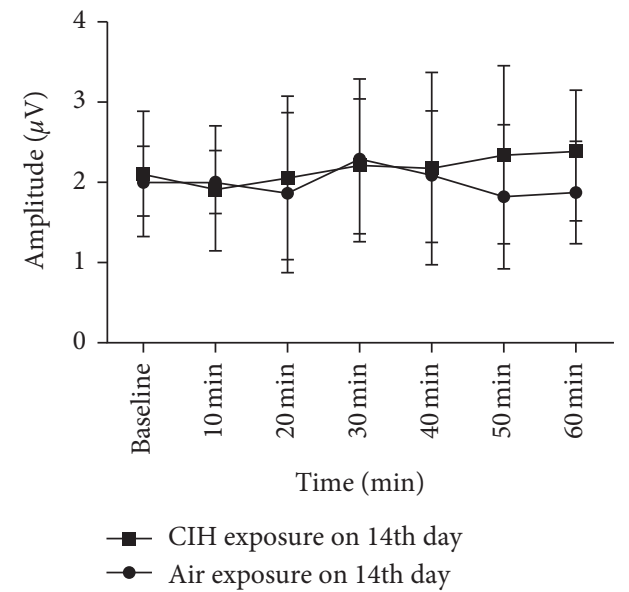

(d)

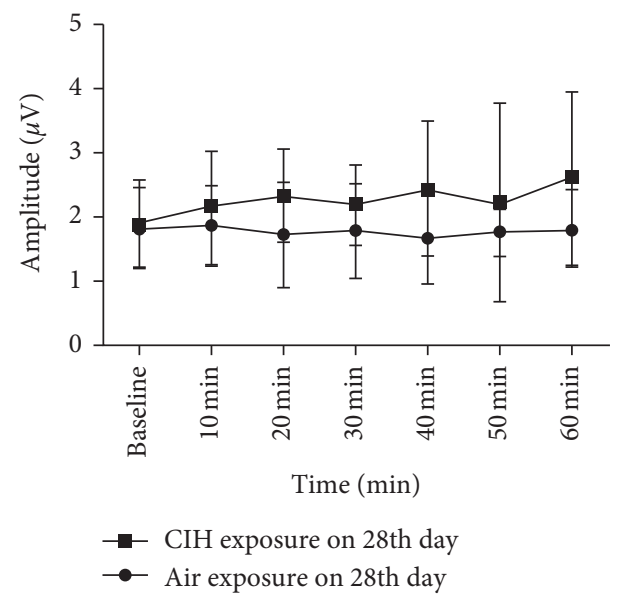

(f)

Figure 3: The comparison of TMS amplitude at different time points after IH stimulation among different groups. (a-f) Values obtained on the 1st, 3rd, 7th, 14th, 21st, and 28th days of the daily air/CIH exposure. ${ }^{*}$ Difference between AIR and CIH groups at the same time point. "Difference at different time points on each day in the $\mathrm{CIH}$ group.

in both CIH-exposed rats and OSAS patients. Edge et al. reported that $\mathrm{CIH}$ caused the respiratory instability by blunting ventilatory LTF in sleeping rats [20]. Thus, it is important to further study the LTF of genioglossus and its central control during sleep. Secondly, the IH protocols described in the previous literature vary considerably in the severity and duration of hypoxic episodes, the interepisode intervals, and the cumulative exposure time. The modest 
low-dose IH protocol we used in this study, hypoxia $(10 \%$ $\mathrm{O}_{2}$ in $\mathrm{N}_{2}$ for $45 \mathrm{~s}$ ) and normoxia $\left(21 \% \mathrm{O}_{2}\right.$ in $\mathrm{N}_{2}$ for $72 \mathrm{~s}$ ) every $180 \mathrm{~s}$ for $8 \mathrm{~h} / \mathrm{d}$, mimics the OSA disease in a moderate severity. It deserves further investigation on the alteration of corticomotor excitability using different IH protocols.

In summary, this study observed the LTF of genioglossus EMG activity and its central control during CIH in awake rats. The genioglossus corticomotor excitability occurred in the first week of $\mathrm{CIH}$ and lasted for three weeks, while the genioglossus EMG activity did not change until the fourth week. Our findings confirmed that $\mathrm{CIH}$ affected the central control of genioglossus, with potential implications for OSAS treatment.

\section{Ethical Approval}

The procedures and experiment protocols were performed in accordance with the National Institutes of Health Guide for Care and Use of Laboratory Animals and were approved by the Animal Ethics and Use Committee of China Medical University.

\section{Disclosure}

This research was first published in ERS as an abstract in 2017, and this is the first time it is published as full text in the Canadian Respiratory Journal.

\section{Conflicts of Interest}

The authors declare that they have no conflicts of interest.

\section{Authors' Contributions}

Ying Zou performed EMG, TMS, data analysis, and manuscript drafting and approved the final version to be published. Xinshi Nie performed EMG and manuscript drafting. Wei Wang and Jian Kang was responsible for study design, data interpretation, critical manuscript revision, and final approval of the version to be published.

\section{Acknowledgments}

This study was supported by the National Natural Science Foundation of China Grant no. 81670085.

\section{Supplementary Materials}

Supplementary Table 1. The value of TMS latency at different time points after IH stimulation among different groups. Supplementary Table 2. The value of TMS amplitude at different time points after IH stimulation among different groups. Supplementary Table 3. The value of genioglossus EMG activity at different time points after IH stimulation among different groups. Supplementary Figure 1. The oxygen concentration of oxycycler. Supplementary Figure 2. TMS stimulation site. (Supplementary Materials)

\section{References}

[1] T. L. Baker and G. S. Mitchell, "Episodic but not continuous hypoxia elicits long-term facilitation of phrenic motor output in rats," Journal of Physiology, vol. 529, no. 1, pp. 215-219, 2000.

[2] S. Mahamed and G. S. Mitchell, "Is there a link between intermittent hypoxia-induced respiratory plasticity and obstructive sleep apnea?," Experimental Physiology, vol. 92, no. 1, pp. 27-37, 2006.

[3] X. P. Tu, J. J. Zuo, K. Hu, J. Kang, Y. Mei, and N. Wang, "Effect of systemic application of 5-hydroxytryptamine on hypoglossal nerve discharge in anesthetized rats," Journal of Molecular Neuroscience, vol. 57, no. 3, pp. 435-445, 2015.

[4] L. C. McKay, W. A. Janczewski, and J. L. Feldman, "Episodic hypoxia evokes long-term facilitation of genioglossus muscle activity in neonatal rats," Journal of Physiology, vol. 557, no. 1, pp. 13-18, 2004.

[5] M. El-Chami, S. Sudan, H. S. Lin, and J. H. Mateika, "Exposure to intermittent hypoxia and sustained hypercapnia reduces therapeutic CPAP in participants with obstructive sleep apnea," Journal of Applied Physiology, vol. 123, no. 4, pp. 993-1002, 1985.

[6] F. L. Powell, W. K. Milsom, and G. S. Mitchell, "Time domains of the hypoxic ventilatory response," Respiration Physiology, vol. 112, no. 2, pp. 123-134, 1998.

[7] F. Sériès, W. Wang, and C. Mélot, "Concomitant responses of upper airway stabilizing muscles to transcranial magnetic stimulation in normal men," Experimental Physiology, vol. 93, no. 4, pp. 496-502, 2008.

[8] F. Sériès, W. Wang, and T. Similowski, "Corticomotor control of the genioglossus in awake OSAS patients: a transcranial magnetic stimulation study," Respiratory Research, vol. 10, no. 1, p. 74, 2009.

[9] J. Su, W. Wang, L. F. Sun, T. Li, D. L. Kong, and J. Kang, "Raphe serotonergic neurons modulate genioglossus corticomotor activity in intermittent hypoxic rats," Respiratory Research, vol. 15, p. 76, 2014.

[10] X. P. Tu, N. Wang, and K. Hu, "The influence of intermittent hypoxia on long-term facilitation of hypoglossal nerve discharge in spontaneous breathing rats," Chinese Journal of Tuberculosis and Respiratory Diseases, vol. 36, no. 6, 2013.

[11] X. S. Nie, L. Zhou, A. D. Wang et al., "Noradrenergic activation of hypoglossal nucleus modulates the central regulation of genioglossus in chronic intermittent hypoxic rats," Frontiers in Neurology, vol. 8, p. 171, 2017.

[12] P. M. Suratt, R. F. McTier, and S. C. Wilhoit, "Upper airway muscle activation is augmented in patients with obstructive sleep apnea compared with that in normal subjects," American Review of Respiratory Disease, vol. 137, no. 4, pp. 889894, 1988.

[13] J. J. Klawe and M. Tafil-Klawe, "Age-related response of the genioglossus muscle EMG-activity to hypoxia in humans," Journal of Physiology and Pharmacology, vol. 54, pp. 14-19, 2003.

[14] R. Moss, "Respiratory responses to single and episodic hypoxia during development: mechanisms of adaptation," Respiration Physiology, vol. 121, no. 2-3, pp. 185-197, 2000.

[15] X. M. Yu, X. Lan, and K. Hu, "Regulation of orexin A on longterm facilitation of genioglossus in rats," Medical Journal of Wuhan University, vol. 36, pp. 853-856, 2015.

[16] J. Pirnar, B. Sirok, and A. Bombac, "Effect of airway surface liquid on the forces on the pharyngeal wall: experimental fluid-structure interaction study," Journal of Biomechanics, vol. 63, pp. 117-124, 2017. 
[17] G. B. Mwenge, M. Friedman, and O. Jacobowitz, "Targeted hypoglossal nerve stimulation for the treatment of obstructive sleep apnea: six-month results," Laryngoscope, vol. 126, no. 11, pp. 2618-2623, 2016.

[18] M. Behan, A. E. Moeser, and C. F. Thomas, "The effect of tongue exercise on serotonergic input to the hypoglossal nucleus in young and old rats," Journal of Speech Language and Hearing Research, vol. 55, no. 3, pp. 919-929, 2012.

[19] J. Huang, S. J. Pinto, H. Yuan et al., "Upper airway collapsibility and genioglossus activity in adolescents during sleep," Sleep, vol. 35, no. 10, pp. 11345-1352, 2012.

[20] D. Edge and K. D. O'Halloran, "Chronic intermittent hypoxia blunts the expression of ventilatory long term facilitation in sleeping rats," Advances in Experimental Medicine and Biology, vol. 860, pp. 335-342, 2015. 


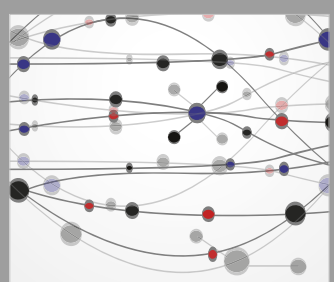

The Scientific World Journal
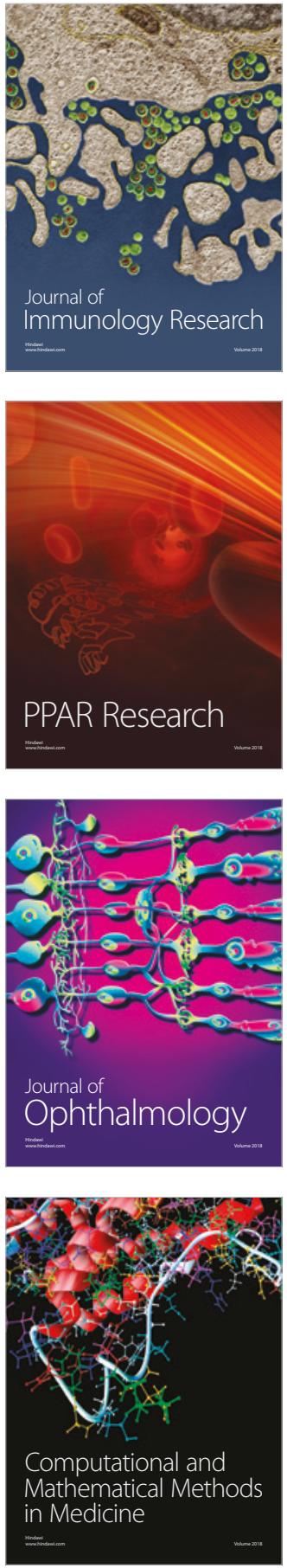

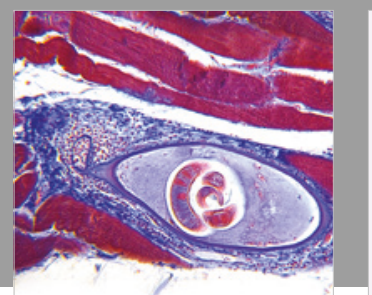

Gastroenterology Research and Practice

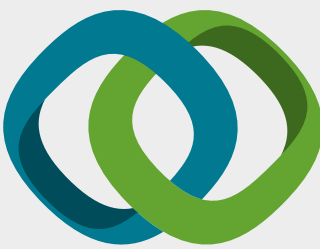

\section{Hindawi}

Submit your manuscripts at

www.hindawi.com
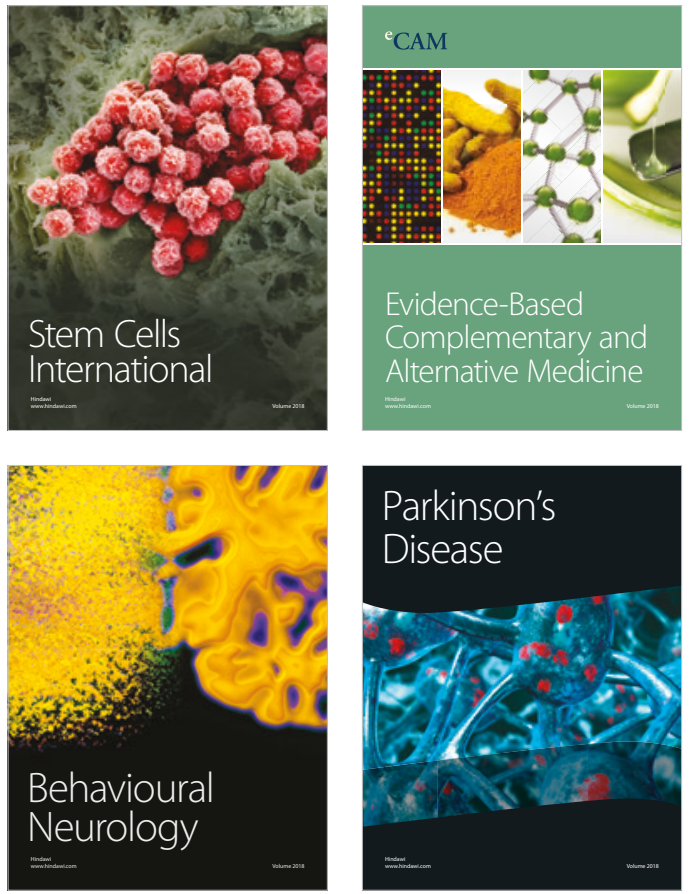

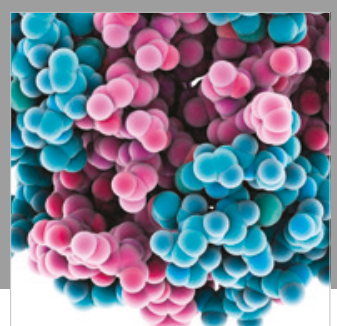

ournal of

Diabetes Research

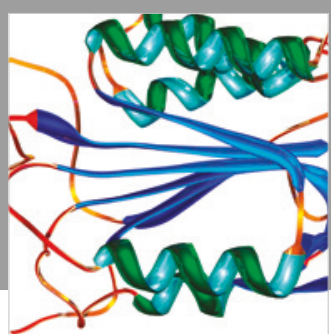

Disease Markers
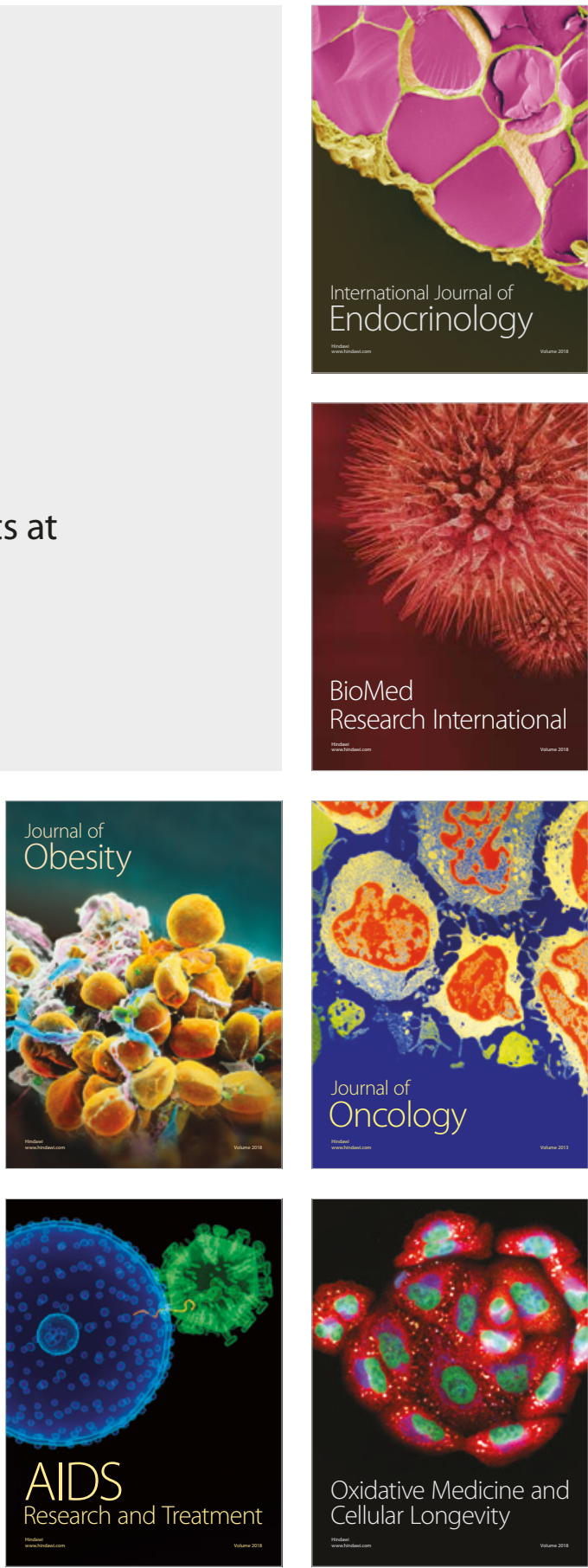